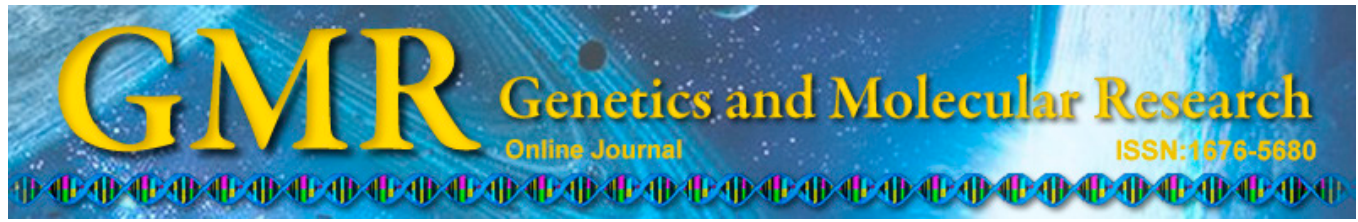

\title{
Effect of age on the immune system and pathology of mice with chronic graft-versus-host disease lupus nephritis
}

\author{
X.F. Ma, F.L. Xu, L.F. Gao, Y.X. Wang and Z.B. Pan \\ The Immunology and Rheumatology Department, \\ Affiliated Hospital of Weifang Medical University, Weifang, \\ Shandong Province, China \\ Corresponding author: F.L. Xu \\ E-mail: mxfwjp188@163.com
}

Genet. Mol. Res. 14 (3): 10999-11005 (2015)

Received January 14, 2015

Accepted May 18, 2015

Published September 21, 2015

DOI http://dx.doi.org/10.4238/2015.September.21.12

\begin{abstract}
We investigated the effect of age on the expression of immune molecules [ANA, C4, double stranded DNA (dsDNA), CD16/32, CD19, CD3, and CD64], urine protein, and pathology in mice with chronic graft-versus-host disease (cGVHD) lupus nephritis (LN), and their relationship with reactivity index score. Mouse models of cGVHD LN were established, and mice were randomly divided into four aged-based groups of nine mice each. Serum levels of ANA, C4, and dsDNA were determined, the urine protein levels were assessed, and expression levels of CD16/32, CD19, CD3, and CD64 were measured. Expression levels of CD16/32+CD19(T1), CD16/CD32+CD3(T2), and CD64+CD3 or CD19(T3) were defined in the thymus, in bone marrow they were defined as CD16/32+CD19(B1), CD16/32+CD3(B2), CD64+CD3 or CD19(B3), and in spleen they were defined as CD16/32+CD19(P1), CD16/32+CD3(P2), CD64+CD3 or CD19(P3), respectively. There were significant differences in the levels of dsDNA and urine protein among the four groups $(\mathrm{P}<0.05)$, which were negatively correlated with age. B1, B2, S1, and S2 were significantly
\end{abstract}


different among the four groups $(\mathrm{P}<0.05)$, with a positive correlation with age for B1 and B2. There was no correlation of expression of ANA, C4, dsDNA, T1-T3, B1-B3, S2-S3 with reactivity index score; S1 was the exception $(r=-0.440, P=0.011)$. Age influenced levels of dsDNA and urine protein in the mouse cGVHD model of LN. S1 was associated with reactivity index score and might also affect pathological changes.

Key words: Lupus nephritis; Immunology; Reactivity index score; Pathology

\section{INTRODUCTION}

With the development of systemic lupus erythematosus (SLE), lupus nephritis (LN) has been reported as one of the most important complications; it affects the prognosis of SLE and makes a major contribution to disability and mortality in the disease (Lenz and Contreras, 2004; Weening et al., 2004; D'Cruz et al., 2007; Ruiz Irastorza et al., 2012; Yap et al., 2012; Zubair and Frieri, 2013). Early presentation of kidney injury occurring in patients with $\mathrm{LN}$ is an abnormal urine protein level. The condition is further characterized by high degrees of albuminuria and hematuria, varieties of cylindruria, and elevated levels of serum creatinine and urea nitrogen. The pathogenesis of LN is very complicated, with several factors involved, such as cytokines, antibodies, and complement proteins. Previous studies have shown that albuminuria is observed in mice 4 weeks after LN is established, and glomerular mesangial segmental, diffuse hyperplasia, global glomerulosclerosis, and pathology changes of the lupus kidney are seen at 12-14 weeks. LN-related immune markers such as ANA, C4, double-stranded DNA (dsDNA), CD16/32, CD19, CD3, and CD64 change during LN pathogenesis (Pérez de Lema et al., 2001; Messmer et al., 2004; Aringer and Smolen, 2005; Liu et al., 2011). However, whether age affects the changes in ANA, C4, dsDNA, pathology, or urine protein, and whether these changes are associated with reactivity index score, remain poorly understood. In this study, we established a chronic graft-versus-host disease (cGVHD) LN mouse model to evaluate the effect of age on the changes in ANA, C4, dsDNA, CD16/32, CD19, CD3, CD64, urine protein, and pathology, as well as the relationship between these changes and reactivity index score, with the aim of finding LN progression-related markers. The development or progression of $\mathrm{LN}$ in patients could be monitored by measuring the changes in certain relevant markers.

\section{MATERIAL AND METHODS}

\section{Reagents}

Anti-CD16/32, anti-CD64, and anti-CD3 antibodies were purchased from BD Biosciences (San Jose, CA, USA). The anti-CD19 antibody was from Beckman Coulter company (USA). The enzyme-linked immunosorbent assay kits for measuring levels of dsDNA, ANA, and $\mathrm{C} 4$ were from Cell Signal Mice.

Male inbred C57BL/6J and DBM2 mice were purchased from the Animal Center, the Affiliated Hospital of Weifang Medical University. The mice were housed in enclosed and sterilized cages. The cGVHD LN mouse model was established as previously described (Bruijn et al., 1988). The mice were randomly divided into four groups of nine mice each 
based on age: group A mice were 8 weeks old; group B were 10 weeks old; group C were 12 weeks old; and group D were 20 weeks old. During the study, two mice each died in groups A and $\mathrm{C}$, and one mouse died in group D.

At the end of the study, mice were anaesthetized with $2.5 \%$ chloral hydrate and incisions were made in their abdomens. Venous blood was drawn from the inferior vena cava and spun to obtain plasma. The kidney, thymus, bone marrow, and spleen were isolated to measure the expression levels of CD16/32, CD19, CD3, and CD64. In addition, paraffin sections of each organ were prepared for hematoxylin and eosin staining and electron microscope analysis.

\section{Measurement of urine protein}

Expression levels of urine protein were measured using an automatic biochemical analyzer from Hitachi company (Japan) according to manufacturer instructions.

\section{Histology and pathology analysis}

Paraffin sections were made from the mouse kidneys. They were dewaxed with xylene and dehydrated by gradient ethanol, then stained using hematoxylin and eosin. The pathology changes of the mouse kidneys were observed under an optical microscope. Pathology scores were assessed according to the 2004 World Health Organization guidelines on kidney pathology (Weening et al., 2004; Medina et al., 2014), which are as follows: 1 for cell hyperplasia; 2 for fibrinoid necrosis and karyorrhexis; 3 for cellular crescents; 4 for agglutinative thrombus and wire loop lesion; and 5 for cell infiltration, renal tubule lesion, and nuclear cell infiltration.

\section{Measurement of ANA, dsDNA, and C4}

Expression levels of ANA, dsDNA, and C4 were measured using ELISA kit [Mouse Anti-dsDNA IgG(total)] from Empire Genomics company (USA) according to manufacturer instructions.

\section{Flow cytometry analysis}

The expression levels of CD16/32, CD19, CD3, and CD64 were measured by flow cytometry. Cells isolated from the thymus, bone marrow, or spleen were washed once in phosphate-buffered saline (PBS) and spun at 1500 revolutions per minute (rpm), then resuspended in PBS to produce a final concentration of $1-2 \times 10^{6}$ cells $/ \mathrm{mL}$. Aliquots of the resuspended cells $(50 \mu \mathrm{L})$ were added to two flow tubes. CD3-peridinin chlorophyll protein, CD19-phycoerythrin (PE), and CD16/CD32-fluorescein isothiocyanate (FITC) (5 $\mu \mathrm{L}$ each) were added to one tube, and CD64-PE $(5 \mu \mathrm{L})$ was added to the other tube. Isotype antibodies were used as a negative control. The tubes containing the antibodies were incubated for 15-30 min in the dark. At the endpoint, PBS was added to wash the cells while spinning. The cell pellets were then resuspended in $500 \mathrm{~mL}$ PBS and analyzed directly by flow cytometry. Expression levels of CD16/32+CD19, CD16/CD32+CD3, and CD64+(CD3 or CD19) in the thymus, bone marrow, or spleen were defined as (T1, T2, T3), (B1, B2, B3), and (S1, S2, S3), respectively. 


\section{Statistical analysis}

A one-sample K-S nonparametric test was used to evaluate whether the data fit a normal distribution in these groups. If they did, one-way analysis of variance was used; if they did not, a $\mathrm{K}$ independent-sample nonparametric test was used. Least significant difference or the Dunnett's T3 test was used for comparison between two groups for homogeneity or heterogeneity of variance, respectively. Correlation of ANA, C4, dsDNA, urine protein, T1, T2, T3, B1, B2, B3, S1, S2, or S3 with reactivity index score was quantified by Spearman correlation coefficient analysis.

\section{RESULTS}

\section{Phenotype}

Increased cell proliferation was observed in the mouse kidneys. Glomerular sclerosis with glomerulus surface adhesion was found in one mouse in group B, whereas in group D, glomerulus surface adhesion (in one mouse), glomerular sclerosis (in one mouse), focal segmental sclerosis (in two mice), and focal segmental and glomerular sclerosis (in one mouse) were seen. Statistical analyses of immune-related and pathological markers for all groups of mice are shown in Tables 1 and 2.

Table 1. Expression levels of ANA, C4, double stranded DNA (dsDNA), and urine protein (means \pm standard
deviation).
\begin{tabular}{lccccc}
\hline Group & N & ANA & C4 & dsDNA & Urine protein \\
\hline A & 7 & $1.14 \pm 1.08$ & $1436.74 \pm 22.83$ & $0.00 \pm 0.44$ & $1.83 \pm 0.67$ \\
B & 9 & $0.45 \pm 0.61$ & $1401.8 \pm 138.56$ & $-0.03 \pm 0.36$ & $1.55 \pm 0.45$ \\
C & 7 & $0.33 \pm 0.51$ & $1443.79 \pm 29.50$ & $-0.39 \pm 0.16$ & $1.22 \pm 0.48$ \\
D & 8 & $0.57 \pm 0.71$ & $1424.46 \pm 26.26$ & $-0.79 \pm 0.21$ & $0.33 \pm 0.36$ \\
F value & & & & & 11.004 \\
P value & & 1.617 & 0.445 & 0.000 & 13.454 \\
& 0.209 & 0.723 & 0.000 \\
\hline
\end{tabular}

Table 2. Expression levels of $\mathrm{CD} 16, \mathrm{CD} 32, \mathrm{CD} 19$, and $\mathrm{CD} 3$ in the thymus and bone marrow (means \pm standard deviation).

\begin{tabular}{lcccc}
\hline Group & N & T1 & T2 & B2 \\
\hline A & 7 & $0.003 \pm 0.005$ & $0.100 \pm 0.098$ & $0.377 \pm 0.423$ \\
B & 9 & $0.007 \pm 0.013$ & $0.016 \pm 0.022$ & $0.022 \pm 0.032$ \\
C & 7 & $0.001 \pm 0.004$ & $0.019 \pm 0.018$ & $0.104 \pm 0.112$ \\
D & 8 & $5.038 \pm 8.691$ & $0.500 \pm 0.773$ & $1.175 \pm 1.617$ \\
F value & & 2.560 & 2.707 & 3.336 \\
P value & & 0.076 & 0.065 & 0.034 \\
\hline
\end{tabular}

\section{Normal distribution assessment}

The levels of ANA, C4, dsDNA, urine protein, T1, T2, and B2 in the different groups all fell within a normal distribution $(\mathrm{P}>0.05)$, whereas the reactivity index scores for $\mathrm{T} 3, \mathrm{~B} 1$, $\mathrm{B} 3, \mathrm{~S} 1, \mathrm{~S} 2$, and $\mathrm{S} 3$ did not $(\mathrm{P}<0.05)$. Statistical analysis of serum immune-related markers and urine protein levels are shown in Tables 1 and 2. 
Except for the comparison between groups A and B, comparison of the dsDNA levels between groups demonstrated a significant difference $(\mathrm{P}<0.05)$. Regarding the urine protein level, there was also a significant difference between groups $(\mathrm{P}<0.05)$ except for groups $\mathrm{A}$ and $\mathrm{B}$, and groups $\mathrm{B}$ and $\mathrm{C}(\mathrm{P}>0.05)$. As seen in Tables 1 and 2 , there was a negative correlation between age and levels of both dsDNA and urine protein. Significant differences in B2, which is the expression of CD16/CD32 and CD3 in bone marrow, were observed between the different groups, and there was a positive correlation with age.

Analysis of the levels of reactivity index score in T3, B1, B3, S1, S2, and S3 revealed significant differences in $\mathrm{B} 1, \mathrm{~S} 1$, and $\mathrm{S} 2$ among the different groups $(\mathrm{P}<0.05)$, demonstrating that age influences the expression of CD16/32+CD19 in the bone marrow and the spleen, and of $\mathrm{CD} 16 / 32+\mathrm{CD} 3$ in the spleen (Table 3).

Table 3. Analysis of the expression of CD16, CD32, CD19, and CD3 in the thymus, bone marrow, and spleen.

\begin{tabular}{lccrrrrr}
\hline & Reactivity index score & T3 & B1 & B3 & S1 & S2 & S3 \\
\hline$\chi^{2}$-value & 3.501 & 2.875 & 20.990 & 0.000 & 11.454 & 26.631 & 0.000 \\
P value & 0.321 & 0.411 & 0.000 & 1.000 & 0.010 & 0.000 & 1.000 \\
\hline
\end{tabular}

\section{Correlative analysis of reactivity index score}

No correlation between levels of ANA, C4, dsDNA, or urine protein with reactivity index score was observed $(\mathrm{P}>0.05)$ (Table 4$)$. However, expression of CD16/32+CD19 in the spleen (S1) was found to be positively correlated with reactivity index score $(\mathrm{r}=-0.440, \mathrm{P}=$ 0.011 ). Expression of these molecules in other organs was not correlated with reactivity index score $(\mathrm{P}>0.05)$.

Table 4. Correlative analysis of reactivity index score with other parameters.

\begin{tabular}{|c|c|c|c|c|c|c|c|c|c|c|c|}
\hline & ANA & $\mathrm{C} 4$ & dsDNA & UP & $\mathrm{T} 1$ & $\mathrm{~T} 2$ & $\mathrm{~T} 3$ & B1 & B2 & S1 & S2 \\
\hline r-value & -0.288 & -0.112 & -0.119 & -0.238 & -0.090 & -0.270 & 0.0384 & -0.005 & 0.039 & -0.440 & -0.123 \\
\hline$P$ value & 0.116 & 0.549 & 0.524 & 0.917 & 0.629 & 0.142 & 0.837 & 0.979 & 0.825 & 0.011 & 0.509 \\
\hline
\end{tabular}

UP: urine protein.

\section{DISCUSSION}

SLE is an autoimmune disease or autoimmune connective tissue disease that can affect any part of the body. LN is a common and severe complication, which is characterized by damage to the glomerulus, vasculature, kidney tubules, and mesenchyme. With the progression of $\mathrm{LN}$, a large amount of urine protein, hematuresis, and cylindruria are observed in patients, with further renal fibrosis or failure eventually developing (Li et al., 2006; Petermann and Floege, 2007).

In this study, we demonstrated increased cell proliferation in the kidneys of mice of different ages, with glomerular sclerosis occurring in 10-week-old mice, and glomerulus surface adhesion, sclerosis, focal segmental sclerosis, and focal segmental with glomerular sclerosis occurring in 20-week-old mice. Through correlative analysis of the reactivity index score, which was based on pathological changes, with the levels of ANA, C4, dsDNA, urine protein, or expression of $\mathrm{CD} 16 / 32, \mathrm{CD} 3, \mathrm{CD} 19$, or CD64 in the thymus, bone marrow, or 
spleen, we found that only the expression of CD16/32+CD19 in the spleen (S1) was correlated with reactivity index score $(\mathrm{P}<0.05)$. This suggests that the pathological changes in the kidneys from mice with $\mathrm{LN}$ could be used as an indicator to evaluate LN progression. Owing to its correlation with pathological changes, expression of CD16/32+CD19 in the spleen might influence disease progression.

Since SLE is systemic, its clinical manifestations are very complicated. It is characterized by the production of autoantibodies against nuclear components; anti-dsDNA antibodies, especially IgG type antibodies with high affinity, are the hallmarks of SLE and play a critical role in its pathogenesis. Previous studies have shown that anti-dsDNA antibody can be purified from the kidneys of LN patients in an active state, implying the role of this antibody in the initiation of organ damage. The severity of kidney disease in SLE patients is usually associated with anti-dsDNA antibody titer. Levels of autoantibodies and cytokines change during the development of LN (Gröndal et al., 2000; Reveille, 2004; Simón et al., 2004; Tucci et al., 2005; Kang and Datta, 2006; Moura et al., 2009). Patients' conditions may progress with age. However, whether autoantibodies and cytokines also change with age remains unclear and requires further investigation. In this study, we found that levels of dsDNA and urine protein differed significantly among the different groups, with negative correlation with age. In addition, significant differences in the expression levels of CD16/32+CD19 in bone marrow (B1) and the spleen (S1), and CD16/32+CD3 in the spleen (S2) were observed in the different groups. Based on the results obtained from this study, the levels of dsDNA and urine protein, and the expression levels of CD16/32+CD19 in the bone marrow (B1) and spleen (S1), and $\mathrm{CD} 16 / 32+\mathrm{CD} 3$ in the spleen (S2) might be used to evaluate LN progression.

\section{REFERENCES}

Aringer M and Smolen JS (2005). Cytokine expression in lupus kidneys. Lupus 14: 13-18.

Bruijn JA, van Elven EH, Hogendoorn PC, Corver WE, et al. (1988). Murine chronic graft-versus-host disease as a model for lupus nephritis. Am. J. Pathol. 130: 639-641.

D’Cruz DP, Khamashta MA and Hughes GR (2007). Systemic lupus erythematosus. Lancet 369: 587-596.

Gröndal G, Gunnarsson I, Rönnelid J, Rogberg S, et al. (2000). Cytokine production, serum levels and disease activity in systemic lupus erythematosus. Clin. Exp. Rheumatol. 18: 565-570.

Kang HK and Datta SK (2006). Regulatory T cells in lupus. Int. Rev. Immunol. 25: 5-25.

Lenz O and Contreras G (2004). Treatment options for severe lupus nephritis. Arch. Immunol. Ther. Exp. 52: 356-365.

Li Y, Tucci M, Narain S, Barnes EV, et al. (2006). Urinary biomarkers in lupus nephritis. Autoimmun. Rev. 5: 383-388.

Liu ZC, Zhou QL, Li XZ, Yang JH, et al. (2011). Elevation of human tumor necrosis factor-like weak inducer of apoptosis in peripheral blood mononuclear cells is correlated with disease activity and lupus nephritis in patients with systemic lupus erythematosus. Cytokine 53: 295-300.

Medina G, González-Pérez D, Vázquez-Juárez C, Sánchez-Uribe M, et al. (2014). Fulminant systemic vasculitis in systemic lupus erythematosus. Case report and review of the literature. Lupus 23: 1426-1429.

Messmer D, Yang H, Telusma G, Knoll F, et al. (2004). High mobility group box protein 1: an endogenous signal for dendritic cell maturation and Th1 polarization. J. Immunol. 173: 307-313.

Moura CG, Lima I, Barbosa L, Athanazio D, et al. (2009). Anti-C1q antibodies: association with nephritis and disease activity in systemic lupus erythematosus. J. Clin. Lab. Anal. 23: 19-23.

Pérez de Lema G, Maier H, Nieto E, Vielhauer V, et al. (2001). Chemokine expression precedes inflammatory cell infiltration and chemokine receptor and cytokine expression during the initiation of murine lupus nephritis. J. Am. Soc. Nephrol. 12: 1369-1382.

Petermann A and Floege J (2007). Podocyte damage resulting in podocyturia: a potential diagnostic marker to assess glomerular disease activity. Nephron. Clin. Pract. 106: c61-c66.

Reveille JD (2004). Predictive value of autoantibodies for activity of systemic lupus erythematosus. Lupus 13: 290-297.

Ruiz Irastorza G, Espinosa G, Frutos MA, Jiménez Alonso J, et al. (2012). Diagnosis and treatment of lupus nephritis. 
Consensus document from the systemic auto-immune disease group (GEAS) of the Spanish Society of Internal Medicine (SEMI) and Spanish Society of Nephrology (S.E.N.). Nefrologia 32: 1-35.

Simón JA, Cabiedes J, Ortiz E, Alcocer-Varela J, et al. (2004). Anti-nucleosome antibodies in patients with systemic lupus erythematosus of recent onset. Potential utility as a diagnostic tool and disease activity marker. Rheumatology 43: 220-224.

Tucci M, Calvani N, Richards HB, Quatraro C, et al. (2005). The interplay of chemokines and dendritic cells in the pathogenesis of lupus nephritis. Ann. N. Y. Acad. Sci. 1051: 421-432.

Weening JJ, D'Agati VD, Schwartz MM, Seshan SV, et al. (2004). The classification of glomerulonephritis in systemic lupus erythematosus revisited. Kidney Int. 65: 521-530.

Yap DY, Tang CS, Ma MK, Lam MF, et al. (2012). Survival analysis and causes of mortality in patients with lupus nephritis. Nephrol. Dial. Transplant. 27: 3248-3254.

Zubair A and Frieri M (2013). Lupus nephritis: review of the literature. Curr. Allergy Asthma Rep. 13: 580-586. 